\title{
The role of head-centric spatial reference with a static and kinetic visual disturbance
}

\author{
MICHEL GUERRAZ \\ Université Pierre Mendès France, Grenoble, France \\ DIDIER POQUIN \\ Centre de Recherches du Service de Santé des Armées, La Tronche, France \\ and \\ THÉOPHILE OHLMANN \\ Université Pierre Mendès France, Grenoble, France
}

\begin{abstract}
A static or kinetic visual disturbance affects subjects' ability to estimate the direction of the gravitational vertical. This kind of error is increased by a head roll inclination. In two experiments, we combined head orientation with a static (Experiment 1: tilted frame) versus kinetic (Experiment 2: rotating disk) visual disturbance. The results showed that with a static visual disturbance, the increase of errors in the inclined head condition was mainly the consequence of a postural head effect like an Aubert effect. On the contrary, with a kinetic visual disturbance, it appears that the disk effect increases with head inclination. However, individual errors observed with the head inclined in front of a stationary disk were systematically correlated with the errors triggered by the same head inclination in front of a rotating disk. These observations confirm that the head axis spatial reference plays an important role in orientation perception, whatever the head position and the kind of visual display.
\end{abstract}

The subjective visual (SV) of tilted observers with tilted head in front of a static sor kinetic visual disturbance has been shown to deviate from the gravitational vertical more than that of observers with head upright. This increase is generally believed to be due to the increase of the weighting of the visual influence, despite the fact that head inclination should produce its own effects. The main aim of the paper was to break down and mutually compare the relative effects of head tilt and visual disturbance on the SV.

\section{Aubert and Müller Effects}

In darkness, tilts of the head and body are well known to induce constant errors in SV that are displaced either in the direction of postural inclination (the Aubert, or A effect) or in the opposite direction (the Müller, or E effect). Many authors (for a review, see Howard, 1986) have suggested that the $\mathrm{E}$ effect is characteristic of small degrees of body inclination (up to $60^{\circ}$ ), whereas the $A$ effect is observed for greater angles $\left(>60^{\circ}\right)$ of tilt. Consequently, tilting the head alone would be expected to pro-

This work was supported by grants from the University Pierre Mendès France of Grenoble (CNRS) and by the Ministère de la Défense (DRET, Contract 92/150). We are grateful to Elwyn Leek for his help with the translation and to the anonymous reviewers for their helpful comments. Correspondence should be addressed to T. Ohlmann, Université Pierre Mendès France (Grenoble II), Laboratoire de Psychologie Expérimentale, BP 47, 38040 Grenoble Cedex 9, France (e-mail: theophile.ohlmann@upmf-grenoble.fr). duce only an E effect, since in this case, the degree of possible tilt is relatively reduced. However, when the tilt is restricted to the head, results vary from one experiment to another. They are E effects (Day \& Wade, 1969; Wade, 1968, 1969; Witkin \& Asch, 1948a), A effects (Dichgans, Diener, \& Brandt, 1974; Parker, Poston, \& Gulledge, 1983), or occasionally no general effect (DiLorenzo \& Rock, 1982; Merker \& Held, 1981).

Two main interpretations of these $A$ and $E$ effects have been suggested. The first is of a mechanical nature and is based on an assumed decrease in otolith sensitivity when the head is inclined (Howard, 1982; Schöne, 1964; Young, 1984). The second, more recent (and more cognitively oriented) interpretation was originally proposed by Mittelstaedt (1983a, 1983b, 1986, 1991). His computational model assumes that the SV is the product of three vectors: a visual, a gravitational, and an idiotropic vector. The latter is manifested by the tendency, observed in upright subjects, to confound the "zenith" and the "just above the line of the head-to-foot axis." This misperception lets the subject set the vertical toward the body $z$-axis when he/ she is tilted. This kind of error, termed A effect, could indicate that the body $z$-axis serves as an orientational reference frame.

\section{Static and Kinetic Visual Disturbances}

Visual disturbances are a well-known source of errors in the judgment of the SV. A static tilted frame induces an illusion in upright viewers in which a physically vertical rod looks tilted in the opposite direction to the frame. 
To appear upright, the rod must be rotated from the objective vertical toward the tilted frame. The magnitude of this error is known as the "rod and frame effect" (RFE), first investigated by Witkin and Asch (1948b). An analogous effect with a roll-rotating display has been extensively reported (Dichgans, Held, Young, \& Brandt, 1972; Held, Dichgans, \& Bauer, 1975).

Despite their apparent similarity, the effects of static and kinetic visual disturbances must be distinguished. First, with small angular visual size displays, the errors they induce on SV have been correlated (Hughes, 1973) but not with large angular size displays (Spinelli, Antonucci, Goodenough, \& Pizzamiglio, 1991). Second, the speed of a kinetic display seems to modulate the link between static and kinetic displays. Babler and Ebenholtz (1989) have shown that the SV deviation observed in front of a static tilted frame is correlated with the SV deviation in front of a low-frequency oscillatory tilted frame. But the link between these two phenomena was shown to diminish as the rate of frame oscillation increased. Third, optic modification as blur drastically reduces the frame effect (Ebenholtz, 1985) but not the disk effect (Leibowitz, Rodemer, \& Dichgans, 1979). Fourth, Dijkstra, Gielen, and Melis (1992) have shown that when the distance between the scene and the observer is increased, the amplitude of postural sway for a stationary scene is enhanced, but it remains constant for a sinusoidal moving scene. The latter effects could be related to other findings on posture (Amblard, Crémieux, Marchand, \& Carblanc, 1985) that static cues (e.g., stroboscopic light) contribute to the orientation and localization of the body, whereas kinetic cues seem to be more involved in posture stabilization.

Hence, it would appear essential to differentiate, as did Paillard and Amblard (1985), the two types of visual information in perceptual studies centered on the spatial orientation issue, particularly in the case of postural disturbances triggered by a head inclination. Previous research has been restricted either to the observation of the effects of a single visual disturbance associated with a head inclination or to a combination of both types of visual disturbance with the head upright. This experiment allows us to cross in a single design both kinds of visual (static vs. kinetic) and postural (head upright vs. head inclined) disturbance.

\section{Combined Head and Visual Disturbances}

When a sideways inclination of the head is combined with a static or kinetic visual perturbation, subjects' estimations of SV have been found to deviate more from the gravitational vertical than estimations made with a single disturbance - that is, inclined head alone, tilted frame alone, or rotating disk alone (Dichgans et al., 1974; Parker et al., 1983). This increase is generally interpreted solely as an overweighting of the visual display inducing a tendency to disregard postural information when the head is inclined. Actually, with a static tilted frame, Poquin et al. (1995) have shown that when the head is inclined, the observed increase in errors cannot be accounted for by a stronger frame effect but mainly by an orientational head effect. In fact, they have demonstrated that when the two disturbances (head and visual frame) are combined, the algebraic deviation from the SV should be broken down into its two components to derive the specific weight of each disturbance. In this way, Poquin et al. have shown that adjustment errors result from the addition of two simple and independent effects-a visual frame effect and a postural head effect.

The interaction between a head inclination and a kinetic visual disturbance (Dichgans et al., 1974; Merker \& Held, 1981; Parker et al., 1983) appears to be more complex than what occurs with a static tilted frame. On the one hand, the direction of SV errors is always determined by the direction of the rotating visual display and SV error amplitude is increased as a function of the degree of the head inclination. On the other hand, the errors are asymmetrical: That is, they are larger when the head is inclined in an opposite direction to the rotating display than when they share the same direction. This pattern of results indicates the existence of an interaction between the head spatial references and the kinetic visual disturbance. This phenomenon is still interpreted mainly as an increased disk effect (Dichgans et al., 1974).

Finally, since the head orientation seems to give rise to various effects according to the nature of the visual disturbances, we investigated here, by using a within-subjects design, the interaction of the position of head tilt with either a static or a kinetic visual disturbance. For this purpose, the same subjects underwent two experiments. In the first one, head inclination was combined with a static tilted frame, and in the second one, head inclination was combined with a kinetic rotating disk. In both conditions, we predicted that the position of the head should always affect subjects' judgment of the SV.

\section{EXPERIMENT 1}

\section{Method}

Subjects. There were 35 women and 34 men, ages 18 to 22 years. All subjects had normal or corrected-to-normal vision.

Apparatus. The rod and frame test paradigm (RFT; Witkin \& Asch, 1948b) and Oltman's (1968) apparatus were used. In this device, a $20^{\circ}$ retinal-size rod that is adjustable in the midfrontal plane is surrounded (RFT condition) or not (control device) by a $28^{\circ}$ retinal size frame that can be tilted either to the left or to the right.

The subjects were seated upright in front of the device. Their heads were held in place at four points by a chinrest-like device that allowed the head to be positioned either upright or inclined to the left or the right.

Procedure. The subjects were tested in two counterbalanced conditions. The first was an RFT condition (frame tilted sideways $28^{\circ}$ to the left or $28^{\circ}$ to the right) with the head upright or tilted sideways $28^{\circ}$ to the left or $28^{\circ}$ to the right. In the second condition, they underwent a control test in darkness (condition without frame) with the head upright or tilted sideways $28^{\circ}$ to the left or $28^{\circ}$ to the right. There were eight trials per condition and per subject. Between each trial, the subjects were asked to close their eyes.

The starting position of the rod was tilted either $28^{\circ}$ to the left or $28^{\circ}$ to the right. The order of presentation of the head position relative to the frame position was counterbalanced.

In all of the tests, subjects were required to adjust the rod to the gravitational vertical with a joystick. They were allowed to self- 
correct without time limit. Before starting the test, three examples of the vertical were shown: a visual vertical (wall ridges or window frames), a gravitational vertical (falling stone), and a postural vertical (a person standing up). This precaution ensured that all subjects understood correctly what was meant by "vertical."

For each trial, the algebraic deviation from the physical vertical was noted. According to convention, deviations to the left (rod turned counterclockwise) were counted as negative and deviations to the right (clockwise) as positive. Analysis was performed on two measures: (1) Constant error is the average of the algebraic differences between the rod adjustment and the gravitational vertical calculated for each trial. It represents the average magnitude of the response and measures the average direction of the errors. (2) Sensitivity to specific disturbance represents the specific weight of each disturbance and is particularly useful when two disturbances are combined. This factorial decomposition (Poquin et al., 1995), based on Nyborg's (1974) formulae, breaks down the frame effect and head effect as follows:

Frame cue

$=[$ (average of algebraic deviations with frame to the right)

- (average of algebraic deviations with frame to the left)]/2

Head cue

$=[$ (average of algebraic deviations with head to the right)

- (average of algebraic deviations with head to the left)]/2

A positive value indicates that the $\mathrm{SV}$ is perceived as being in the same direction as the perturbation. A negative value indicates the opposite. For both types of measures, an analysis of variance (ANOVA) and a paired-samples $t$ test were used for all mean comparisons, and a Spearman coefficient was used for the correlations. A .05 significance level was adopted throughout.

\section{Results and Discussion}

Constant errors and sensitivity to specific disturbance for all conditions are reported respectively in Tables $\mathrm{A} 1$ and $\mathrm{A} 2$ in the Appendix.

Control condition: Effect of head orientation without frame. Without a visual frame, SV depended on head orientation. Figure 1 shows that when the head was inclined to the left, SV deviated to the left, and when the head was inclined to the right, SV deviated to the right. This effect was significant $[F(2,136)=21.7]$. Adjustments of each head position were significantly different

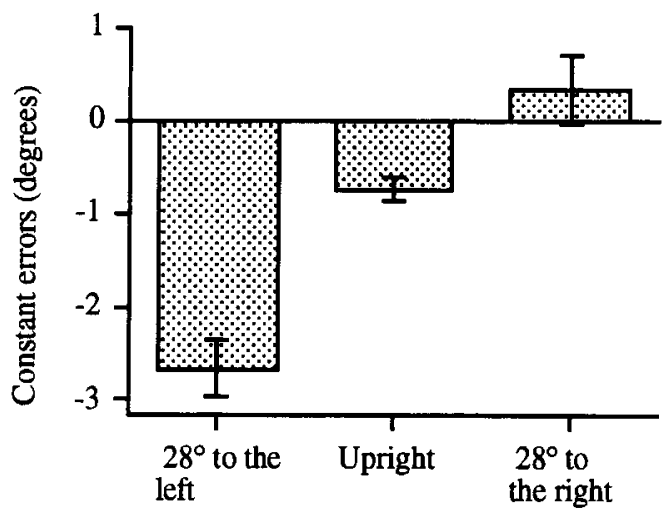

Head orientation

Figure 1. Average of adjustment errors (degrees) in algebraic deviations as a function of head orientation in the control condition without visual disturbance. Error bars are standard errors of the mean. from one another (Newman-Keuls). In addition, SV was estimated significantly to the left of the physical vertical when the head was upright $[t(68)=6.05]$. This slight counterclockwise deviation with head upright has also been reported elsewhere (e.g., Bauermeister, 1964; Dichgans et al., 1974). Figure 1 shows that for a $28^{\circ}$ head inclination, SV was mostly perceived as being in the same direction as the orientation of the head, as expressed by the positive "head cue" $\left(1.49^{\circ} \pm 2.6\right)$. Restricted to the orientation of the head, this phenomenon could be compared to an A effect.

Experimental condition: Effect of head orientation with a visual tilted frame. In the combined condition (Figure 2), each disturbance (head or frame position) attracted SV toward its own orientation. ANOVA indicated a significant effect of the frame factor $[F(1,68)=36.9]$ and a significant effect of the head factor $[F(2,136)=$ 11.1], but no interaction between the two factors $[F(2,136)<1]$. Figure 2 shows that in the combined condition, SV depended on the direction of both the frame and the head, as expressed by the positive "frame cue" $\left(1.49^{\circ} \pm 2.5^{\circ}\right)$ and the positive "head cue" $\left(1.85^{\circ} \pm 4.6^{\circ}\right)$.

The frame cue, computed when the head was inclined, did not differ from its control counterpart, computed when the head was upright $\left(1.68^{\circ} \pm 1.7^{\circ}\right)$, and the two were correlated $[r(69)=.74]$. In the same way, the head cue computed when the frame was tilted did not differ from its control counterpart, which was computed without the frame, and the two were correlated $[r(69)=.52]$. Moreover, the head and frame cues in the combined condition were independent $[r(69)=.18$, n.s. $]$, as in the isolated condition $[r(69)=.20$, n.s. $]$.

Sex differences in frame and head cues. Head and frame cues for women and men are reported in Table A3. The frame cue computed when the head was tilted increased and decreased, respectively, in women $[t(34)=$ $2.2]$ and men $[t(33)=4.9]$ relative to their control counterparts computed with the head upright. However, the two frame cues were correlated in each population [women, $r(35)=.77$; men, $r(34)=.64$ ]. Head cue computed when the frame was tilted remained constant in women $[t(34)<1]$ and men $[t(33)<1]$ relative to the darkness condition. The two head cues remained correlated in each population [women, $r(35)=.51$; men, $r(34)=$ .48]. Hence an analysis of sex differences showed that, despite a slight variation of the frame cue, the head cue remained constant in both male and female subjects.

In summary, Experiment 1 confirmed that with a static visual disturbance, the increase of errors in the inclined head condition was mainly the consequence of a postural head effect like an Aubert effect (Poquin et al., 1995). The same kind of experiment was then carried out with a kinetic visual disturbance.

\section{EXPERIMENT 2}

\section{Method}

Subjects. The 69 subjects who participated in Experiment 1 took part in Experiment 2. 


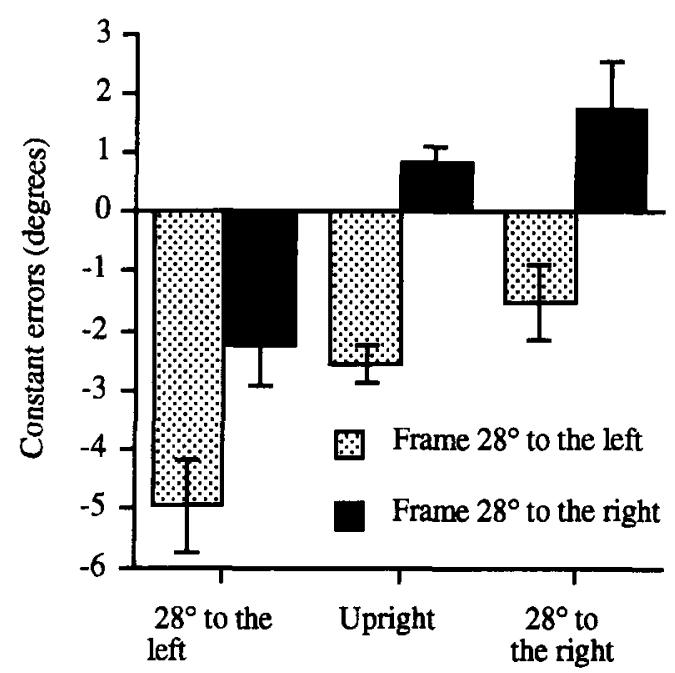

Head orientation

Figure 2. Average of adjustment errors (degrees) in algebraic deviations as a function of head and the frame orientation. Error bars are standard errors of the mean.

Apparatus. Oltman's (1968) portable RFT, used in Experiment 1, was transformed in such a way that a circular disk took the place of the frame. This circular disk, subtending $28^{\circ}$ of visual angle, consisted of irregular speckles on a black background. It could be rotated at $30 \% \mathrm{sec}$ in a clockwise $(\mathrm{CW})$ or counterclockwise $(\mathrm{CCW})$ direction around the subject's line of sight, or could be stationary (control condition). At its center there was a circular black surface without speckles in which an adjustable rod (subtending $10^{\circ}$ visual angle size) was located. In keeping with Witkin and Asch's (1948b) terminology, we have called this device the "rod and disk test" (RDT).

As in Experiment 1, the subjects were seated upright in front of the device. Their heads were held in place at four points by a chinrestlike device that allowed the head to be positioned either upright or tilted sideways to the left or right.

Procedure. The subjects were tested in two counterbalanced conditions. The first condition consisted of the RDT (disk rotation $30 \% \mathrm{sec} \mathrm{CW}$ or $30 \% \mathrm{sec} \mathrm{CCW}$ ) with the head upright or inclined sideways $28^{\circ}$ to the left or $28^{\circ}$ to the right. In the second condition, they underwent a control test (stationary disk) with the head upright or inclined sideways $28^{\circ}$ to the left or $28^{\circ}$ to the right. In this control test, the disk was rotated before each trial in order to prevent any visual cues arising between trials. There were eight trials per condition and per subject. Between each trial, the subjects were asked to close their eyes.

The starting position of the rod was tilted either $28^{\circ}$ to the left or $28^{\circ}$ to the right. The order of presentation of the head position relative to the disk rotation was counterbalanced. To dissipate the tilting and rotating effects, a period of $30 \mathrm{sec}$ of exposure preceded each new condition of measurements and $30 \mathrm{sec}$ of rest with closed eyes followed each condition. In all conditions, the subjects were required to adjust the rod to the gravitational vertical with a joystick. Before starting the test, we repeated the same instructions used in Experiment 1 .

For each trial, the algebraic deviation from the physical vertical was noted. According to convention, deviations to the left (rod turned $\mathrm{CCW}$ ) were counted as negative, and deviations to the right (CW) were counted as positive. As in the first experiment, two types of analysis were computed from the collected data-the constant errors and the sensitivity to specific disturbance. This last was computed by the same factorial decomposition used in the previous experiment:
Disk cue

$=[$ (average of algebraic deviations with disk rotating $\mathrm{CW})$

- (average of algebraic deviations with disk rotating CCW)]/2

Head cue

$=[$ (average of algebraic deviations with head to the right)

- (average of algebraic deviations with head to the left)]/2

A positive value indicates that the $\mathrm{SV}$ is perceived as being in the same direction as the perturbation. A negative value indicates the opposite.

For both types of measures, an ANOVA and a paired-samples $t$ test were used for all mean comparisons, and a Spearman coefficient was used for the correlations. A .05 significance level was adopted throughout.

\section{Results and Discussion}

Constant errors and the sensitivity to specific disturbance for each condition are reported respectively in $\mathrm{Ta}$ bles $\mathrm{A} 1$ and $\mathrm{A} 2$.

Control condition: Effect of head orientation with a stationary disk. When the subjects were viewing a stationary disk, SV depended on head orientation. Figure 3 shows that when the head was inclined to the left, SV deviated to the left, but when the head was inclined to the right, SV slightly deviated to the right. This head orientation effect was significant $[F(2,136)=12.6]$. Only the $28^{\circ}$ left position was different from the others (NewmanKeuls). As in Experiment 1, SV deviated significantly to the left of the physical vertical when the head was upright $[t(68)=3.2]$.

SV deviated toward the orientation of head tilt, as expressed by the positive head cue $\left(1.24^{\circ} \pm 2.9^{\circ}\right)$ computed in the control condition. This cue did not significantly differ from, but was correlated with, the head cue observed in Experiment 1, in which the head was inclined in front of a tilted frame $[r(69)=.52]$ or in darkness $[r(69)=.59]$.

Experimental condition: Effect of head orientation with a rotating display. ANOVA indicated a significant effect of the disk factor $[F(1,68)=216]$, no effect of the

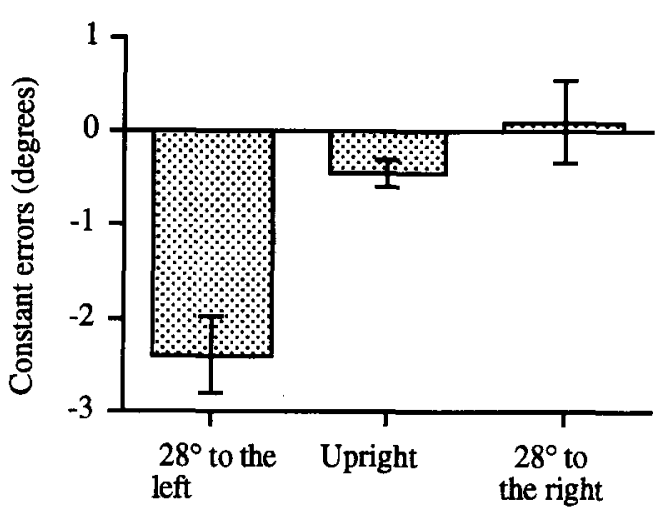

Head orientation

Figure 3. Average of adjustment errors (degrees) in algebraic deviations as a function of head orientation with a stationary disk. Error bars are standard errors of the mean. 
head factor $[F(2,136)<1]$, and an interaction between the two factors $[F(2,136)=44]$. As can be seen in Figure 4 , when the head was upright, SV deviated toward the direction of disk rotation, and this disk effect increased with the inclination of the head. There was no significant difference between the conditions in which the head was inclined to the left or to the right when the disk rotated, respectively, in a CW direction or in a CCW direction (Newman-Keuls). An earlier reported asymmetric effect relative to the side of the head position observed on small samples of subjects (Dichgans et al., 1974; Merker \& Held, 1981) did not seem to occur in our relatively large sample.

This result indicates that the amount of SV deviation observed in the combined condition relied on disk rotation only. However, as can be seen from Figure 5, correlations between the different conditions depended on head orientation and not on the direction of disk rotation. The SV deviation observed when the head was inclined to one side in the stationary disk condition correlated with its counterparts when the disk rotated CCW (Figures 5a, 5b) or CW (Figures 5c, 5d). In the same way, when the head was inclined to one side, we observed small but significant correlations between conditions of opposite disk rotation (Figures 5e, 5f). These positive correlations mean that the direction of head tilt brought into play a common factor that resulted in similar (size and direction) errors in the two conditions. On the contrary, when the head was inclined in opposite directions, there was no correlation between these conditions when the two disks rotated in the same direction (CCW, Figure $5 \mathrm{~g}$; $\mathrm{CW}$, Figure $5 \mathrm{~h}$ ).

Despite the increase in errors in the direction of the disk when the head was inclined, correlation analysis shows



Figure 4. Average of adjustment errors (degrees) in algebraic deviations as a function of head orientation and disk rotation. Error bars are standard errors of the mean. that the individual amplitude of SV error was determined mainly by the direction of the head inclination and not by the direction of disk rotation (e.g., Figures $5 \mathrm{~b}$ and $5 \mathrm{~d}$ ). On the contrary (Figures $5 \mathrm{~g}$ and $5 \mathrm{~h}$ ), when the head was inclined to the opposite side, there was no correlation despite the same disk rotation. These results lead us to conclude that the head orientation effect remained present while the disk effect increased. Does the extraction of sensitivity to each specific disturbance in the combined condition confirm the increased disk effect and validate the hypothesis of head orientation effect constancy?

The disk cue computed with an inclined head $\left(4.79^{\circ} \pm\right.$ $2.7^{\circ}$ ) was different $[t(68)=10.6]$ from its counterpart with the head upright $\left(2.58^{\circ} \pm 1.8^{\circ}\right)$, but the two were highly correlated $[r(69)=.71]$. This observation confirms that the disk effect increased in the combined condition. With the head upright, a weak but significant correlation between the disk cue and the frame cue (Experiment 1) was observed $[r(69)=.25]$.

The head cue computed in the rotating disk condition was highly correlated with its counterpart in the stationary disk condition $[r(69)=.63]$. Moreover, this head cue obtained in the kinetic condition correlated with the head cue observed in darkness $[r(69)=.54]$ and in the tilted frame condition $[r(69)=.52]$, as observed in Experiment 1 . Hence, as seen previously, whatever type of analysis or experimental condition, these results show that the inclined head effect always contributes to the amount of SV estimation error. The head cue and the disk cue were relatively independent both in the combined $[r(69)=-.18, \mathrm{n} . \mathrm{s}$.$] and the isolated conditions$ $[r(69)=.19$, n.s. $]$.

It should be noted that the head cue observed in the rotating condition $\left(-0.05^{\circ} \pm 3.1\right)$ was smaller than its stationary counterpart $[t(68)=4.3]$ and did not differ from the zero. However, a test for the homogeneity of variance with O'Brien's (1981) transformation showed that the dispersion of interindividual differences of head cue is equivalent in the stationary disk and rotating disk conditions $[t(68)=1.1$, n.s. $]$. Consequently, the effect of head tilt orientation did not disappear in front of a rotating display, but it did shift. In the combined condition, SV deviated toward the opposite direction of the head inclination, relative to the SV deviation in the control condition (stationary disk). This shift appeared clearly when results were split between the two groups on the basis of sex.

Sex differences in disk and head cues. Head and disk cues for women and men are reported in Table A3. The disk cue computed when the head was tilted increased in women $[t(34)=8.1]$ and in men $[t(33)=6.8]$ relative to that of their control counterparts computed when the head was upright. The two disk cues were correlated in each population [women, $r(35)=.64$; men, $r(34)=$ $.78]$. On the contrary (Figure 6), head cue computed in the rotating disk condition decreased in women $[t(34)=$ $2.5]$ as well as in men $[t(33)=3.6]$ despite important intersex differences [stationary condition, $t(67)=2.4$; rotating condition, $t(67)=3.14]$. In addition, the two head 


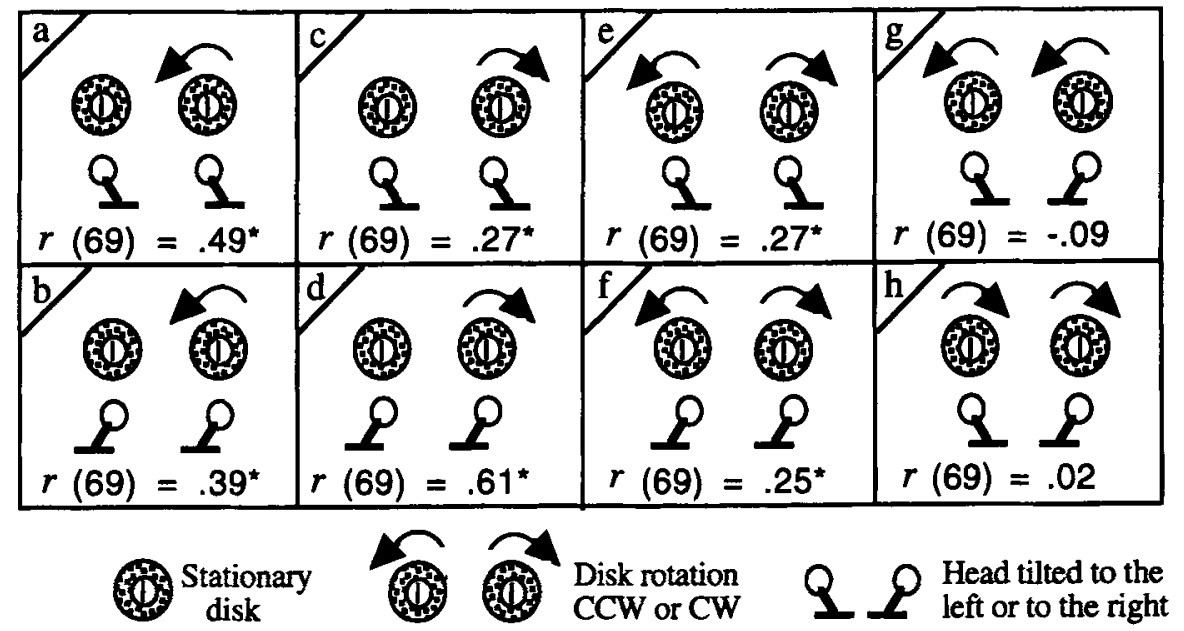

Figure 5. Correlations of constant errors (subjective vertical) between the conditions according to the head tilt and the disk rotation. *Significant correlations.

cues were still correlated in women $[r(35)=.56]$ and men $[r(34)=.61]$.

Thus, in women and men, the tendency to set the SV toward the head orientation decreased more in the rotating condition than in the stationary one. The significant $A$ effect and $\mathrm{E}$ effect observed in the rotating condition in women and men, respectively, cancel each other out for the global population $\left(-0.05^{\circ} \pm 3.1^{\circ}\right)$.

\section{GENERAL DISCUSSION}

When the head is oriented upright, the link between the frame effect and the disk effect is not clearly established. According to Spinelli et al. (1991), the visual angle size of the display is an important factor that determines the correlation between the two phenomena. The small but significant correlation found in our study between frame and disk effect cues can be interpreted as consistent with this observation. The angular size of the displays used in our study $\left(28^{\circ}\right)$ was smaller than that used by Spinelli et al. $\left(150^{\circ}\right.$ disk and $40^{\circ}$ frame) and larger than that $\left(15^{\circ}\right)$ used by Hughes (1973). So, the larger the angular size of the displays is, the more uncorrelated the two phenomena are.

In other respects, it should be noted that despite the strong disparity of our two devices (RFT and RDT), the head orientation effect was present across the different experimental conditions. Indeed, head cues computed in the control conditions (darkness and stationary disk) correlated not only with their experimental counterparts (tilted frame and rotating disk) but also with each other. This head effect was always present in the determination of the SV, but its amplitude varied. In the first experiment, it appears that the well-known increase in adjustment error when the head is inclined in front of a tilted frame was not simply the consequence of a larger visual effect, but of a mere additive inclined head effect (in women as well as men). In Experiment 2, with a rotating display, the effect of head orientation appears to be more complex. With- out crossed analyses involving sex differences and correlations, the global error on SV in this combined condition could be interpreted entirely as a sole visual effect due to the disk rotation. Thus, the maintenance of the correlation between the head cues computed between stationary and kinetic visual conditions suggests the involvement of a head orientation effect.

The larger visual effect with head inclination in front of a kinetic display (Dichgans et al., 1974; Merker \& Held, 1981) has generally been attributed to compensation by visual afference of a putative decline in otolith sensitivity (Bischof, 1974). However, Yardley (1990) did not observe this strong increased disk effect with an inclined subject without proprioceptive input below the neck but with an intact otolith system. Thus, the increased disk effect in the inclined head condition seems to be triggered

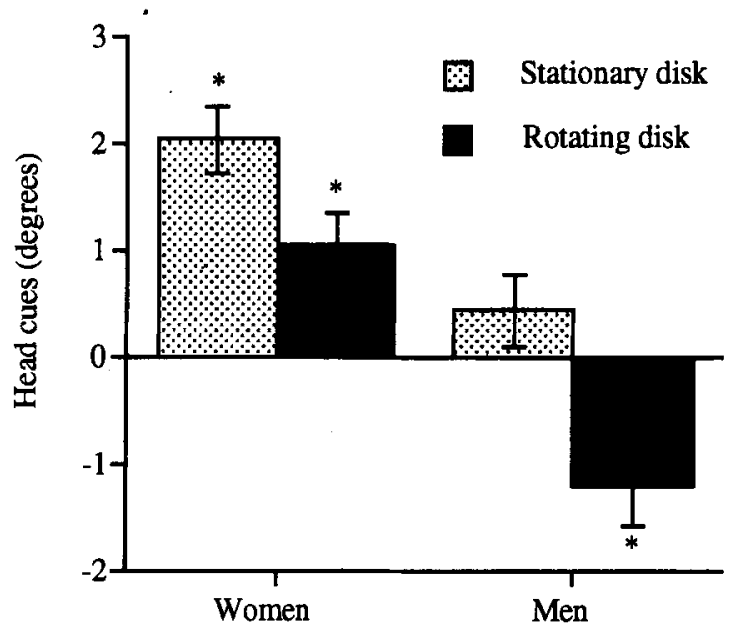

Figure 6. Average of head sensitivity cue (degrees) with a stationary and a rotating disk for women and men. A positive value indicates an $A$ effect whereas a negative value indicates an $E$ effect. *Averages significantly different from zero. Error bars are standard errors of the mean. 
more by somatosensory information than by a simple decrease of otolithic sensitivity. Our results are also difficult to explain by this kind of decrease. First, with a constant head inclination whatever the visual condition, an otolithic decline would have had the same effect and yielded the same increased visual effect. However, head tilt was associated with a large increased visual effect only in the kinetic condition. Second, in keeping with the otolithic sensitivity decline hypothesis, the global error observed with the inclined head and rotating display would have been determined mainly by disk rotation. This was not the case with our results.

The orientational role of the $z$ longitudinal axis (Luyat, Ohlmann, \& Barraud, 1997) or the idiotropic vector (Mittelstaedt, 1983a, 1983b, 1986) appears to be a more interesting alternative explanation of our results than a putative otolithic sensitivity decrease. As noted, in his computational model, Mittelstaedt assumed, on the one hand, that $\mathrm{SV}$ is determined by the combination of three vectors (an idiotropic vector centered on the longitudinal axes of the head and the body, a gravitational vector, and a visual vector). On the other hand, the model predicts, for any given subject, a constant value of the idiotropic vector. However, although our results suggest that the idiotropic vector seems to always be present, it acts in a manner more complex than previously thought.

When the visual field is static, Mittelstaedt's second assumption concerning the stability of the idiotropic vector holds, but kinetic visual disturbance yields more complex results. Hence, the decrease in the A effect observed in Experiment 2 could be reinterpreted as the consequence of an apparent length diminution of head idiotropic vector. This diminution does not fit with Mittelstaedt's model, in which the length of the idiotropic vector is said to be constant. In the kinetic condition, Mittelstaedt (1991) himself suggested a difficulty in referring to the body position and consequently to the idiotropic vector. His suggestion was based on the absence of polarity (up/ down) in the kinetic visual scene as compared with its static counterpart. Therefore the $z$-axis may be an orientational reference highly compatible with a structured visual environment but relatively unavailable in kinetic visual scenes. Actually an indirect argument in favor of the stability of the idiotropic vector attraction is given here by the correlational analysis. Individual differences in subjects' reliance on the head reference remained constant across various tasks; thus the more a subject showed an "idiotropic tendency" in a given situation, the more he/ she reproduced this tendency in another situation. Hence, this stability, despite drastic changes in visual information, confirms the significant role of egocentered reference frames in spatial orientation.

\section{REFERENCES}

amblard, B., Crémieux, J., Marchand, A. R., \& Carblanc, A. (1985). Lateral orientation and stabilisation of human stance: Static versus dynamic visual cues. Experimental Brain Research, 32, 21-37.
BABleR, T. G., \& Ebenholtz, S. M. (1989). Effects of peripheral circular contours on dynamic spatial orientation. Perception \& Psychophysics, 45, 307-314.

BAUERMEISTER, M. (1964). Effect of body tilt on apparent verticality, apparent body position and their relation. Journal of Experimental Psychology, 67, 142-147.

BISCHOF, N. (1974). Optic vestibular orientation to the vertical. In H. H. Kornhuber (Ed.), Handbook of sensory physiology (Vol. 6, Pt. 2, pp. 155-190). New York: Springer-Verlag.

DAY, R. H., \& WADE, N. J. (1969). Mechanisms involved in visual orientation constancy. Psychological Bulletin, 1, 33-42.

Dichgans, J., Diener, H. C., \& BrandT, T. (1974). Optokineticgraviceptive interaction in different head positions. Acta Otolaryngologica, 78, 213-221.

Dichgans, J., Held, R., Young, L. R., \& Brandt, T. (1972). Moving visual scenes influences the apparent direction of gravity. Science, $178,1217-1219$.

Dijkstra, T. M., Gielen, C. C., \& Melis, B. J. (1992). Postural responses to stationary and moving scenes as a function of distance to the scene. Human Movement Science, 11, 195-203.

Dilorenzo, J. R., \& Rock, I. (1982). The rod and frame effect as a function of the righting of the frame. Journal of Experimental Psychology: Human Perception \& Performance, 8, 536-546.

Ebenholtz, S. M. (1985). Blur-modulated orientation perception in the rod-and-frame task. Perception \& Psychophysics, 37, 109-113.

Held, R., DichGaNs, J., \& BaUER, J. (1975). Characteristics of moving visual scenes influencing spatial orientation. Vision Research, 15, 357-365. HOWARD, I. P. (1982). Human visual orientation. London: Wiley.

HOWARD, I. P. (1986). The perception of posture, self motion, and the visual vertical. In K. R. Boff, L. Kaufmann, \& J. P. Thomas (Eds.), Handbook of perception and human performance (pp. 18.1-18.61). New York: Wiley.

Hughes, P. C. (1973). The influence of the visual field upon the visual vertical in relation to ocular torsion of the eye. Dissertation Abstracts International, 33, 4686B.

Leibowitz, H. W., Rodemer, C. S., \& Dichgans, J. (1979). The independence of dynamic spatial orientation from luminance and refractive error. Perception \& Psychophysics, 25, 75-79.

Luyat, M., Ohlmann, T., \& Barraud, P. A. (1997). Subjective vertical and postural activity. Acta Psychologica, 95, 181-193.

MERKER, B. H., \& HeLD, R. (1981). Eye torsion and the apparent horizon under head tilt and visual field rotation. Vision Research, 21, 543-547.

MittelstaedT, H. (1983a). A new solution to the problem of the subjective vertical. Naturwissenschatften, 70, 272-281.

Mitielstaedt, H. (1983b). Towards understanding the flow of information between objective and subjective space. In F. Huber \& H. Markl (Eds.), Neuroethology \& behavioral physiology (pp. 382402). Heidelberg: Springer-Verlag.

Mittelstaedt, H. (1986). The subjective vertical as a function of visual and extraretinal cues. Acta Psychologica, 63, 63-88.

MitTELSTAEDT, H. (1991). The role of the otoliths in perception of the orientation of self and world to the vertical. Zoological Journal of Physiology, 95, 419-425.

Ny BORG, H. (1974). A method for analysing performance in the rod and frame test: I. Scandinavian Journal of Psychology, 15, 119-123.

O'BRIEN, R. G. (1981). A simple test for variance effect in experimental designs. Psychological Bulletin, 89, 570-574.

OltMAN, P. K. (1968). A portable rod-and-frame apparatus. Perceptual \& Motor Skills, 26, 503-506.

Paillard, J., \& Amblard, B. (1985). Static versus kinetic cues for the processing of spatial relationships. In D. J. Ingle, M. Jeannerod, \& D. N. Lee (Eds.), Brain mechanisms and spatial vision (pp. 299-330). The Hague: Nijhoff.

Parker, D. E., Poston, R. L., \& Gull.edge, W. L. (1983). Spatial orientation: Visual-vestibular-somatic interaction. Perception \& Psychophysics, 33, 139-146.

Poquin, D., Guerraz, M., Ohlmann, T., Marendaz, C., Brenet, F., \& RAPHEL, C. (1995). Visual disturbance, head tilt and Aubert effect. Current Psychology of Cognition, 14, 231-253. 
SCHÖNE, H. (1964). On the role of gravity in human spatial orientation. Aerospatial Medicine, 35, 764-772.

Sinelli, D., Antonucci, G., Goodenough, D. R., \& Pizzamiglio, L. (1991). Psychophysiological mechanisms underlying the rod-andframe illusion. In S. Wapner \& J. Demick (Eds.), Bio-psycho-social factors in cognitive style (pp. 37-60). Hillsdale, NJ: Erlbaum.

WADE, N. J. (1968). Visual orientation during and after lateral head, body, and trunk tilt. Perception \& Psychophysics, 3, 215-219.

WADE, N. J. (1969). The effect of stimulus line variations on visual orientation with head upright and tilted. Australian Journal of Psychology, 21, 177-185.

WITKIN, H. A., \& AsCH, S. (1948a). Studies in space orientation:
III. Perception of the upright in the absence of visual field. Journal of Experimental Psychology, 38, 603-614.

WITKIN, H. A., \& AsCH, S. (1948b). Studies in space orientation: IV. Further experiments on perception of the upright with visual fields. Journal of Experimental Psychology, 38, 762-782.

YARDLEY, L. (1990). Contribution of somatosensory information to perception of the visual vertical with body tilt and rotating visual field. Perception \& Psychophysics, 48, 131-134.

YounG, L. R. (1984). Perception of the body in space; mechanisms. In I. Darian Smith (Ed.), Handbook of physiology: Sec. 1. The nervous system: Vol. 3. Sensory processes (Pt., 2, pp. 1023-1066). Bethesda, MD: American Physiological Society.

\section{APPENDIX}

Table A1

Means and Standard Deviations in Degrees of the Constant Error of Discrepancies Between the Rod Adjustment and the Gravitational Vertical for All Conditions in Experiments 1 and 2

\begin{tabular}{|c|c|c|c|c|c|c|}
\hline \multirow[b]{3}{*}{ Condition } & \multicolumn{6}{|c|}{ Head Orientation } \\
\hline & \multicolumn{2}{|c|}{$28^{\circ}$ to the Left } & \multicolumn{2}{|c|}{ Upright $\left(0^{\circ}\right)$} & \multicolumn{2}{|c|}{$28^{\circ}$ to the Righ } \\
\hline & $M$ & $S D$ & $M$ & $S D$ & $M$ & $S D$ \\
\hline \multicolumn{7}{|c|}{ Experiment 1} \\
\hline $\begin{array}{l}\text { No frame (control condition) } \\
\text { Frame } 28^{\circ} \text { to the left } \\
\text { Frame } 28^{\circ} \text { to the right }\end{array}$ & $\begin{array}{l}-2.66 \\
-4.96 \\
-2.24\end{array}$ & $\begin{array}{l}2.7 \\
6.7 \\
5.5\end{array}$ & $\begin{array}{r}-0.74 \\
-2.55 \\
0.81\end{array}$ & $\begin{array}{l}1.1 \\
2.7 \\
2.4\end{array}$ & $\begin{array}{r}0.33 \\
-1.53 \\
1.74\end{array}$ & $\begin{array}{l}3.2 \\
5.3 \\
6.6\end{array}$ \\
\hline \multicolumn{7}{|c|}{ Experiment 2} \\
\hline $\begin{array}{l}\text { Stationary disk (control condition) } \\
\text { Disk rotation } 30 \% \mathrm{sec} \mathrm{CCW} \\
\text { Disk rotation } 30 \% \mathrm{sec} \mathrm{CW}\end{array}$ & $\begin{array}{r}-2.39 \\
6.15 \\
3.29\end{array}$ & $\begin{array}{l}3.5 \\
3.9 \\
5.1\end{array}$ & $\begin{array}{r}-0.45 \\
-3.44 \\
1.72\end{array}$ & $\begin{array}{l}1.2 \\
2.8 \\
2.3\end{array}$ & $\begin{array}{r}0.09 \\
-6.39 \\
3.34\end{array}$ & $\begin{array}{l}3.7 \\
5.5 \\
4.1\end{array}$ \\
\hline
\end{tabular}

Note-The counterclockwise (CCW) deviations of the rod were negative. The clockwise (CW) deviations of the rod were positive.

Table A2

Means and Standard Deviations in Degrees of the Sensitivity to Specific Disturbance Computed in Experiments 1 and 2

\begin{tabular}{|c|c|c|c|c|c|c|c|c|}
\hline \multirow[b]{3}{*}{ Condition } & \multicolumn{4}{|c|}{ Experiment 1} & \multicolumn{4}{|c|}{ Experiment 2} \\
\hline & \multicolumn{2}{|c|}{ Head Cue } & \multicolumn{2}{|c|}{ Frame Cue } & \multicolumn{2}{|c|}{ Head Cue } & \multicolumn{2}{|c|}{ Disk Cue } \\
\hline & $M$ & $S D$ & $M$ & $S D$ & $M$ & $S D$ & $M$ & $S D$ \\
\hline Inclin & 1.49 & 2.6 & & & 1.24 & 2.9 & & \\
\hline Head upright with visual perturbation & & & 1.68 & 1.7 & & & 2.58 & 1.8 \\
\hline Inclined head and visual perturbation & 1.85 & 4.6 & 1.49 & 2.5 & -0.05 & 3.1 & 4.79 & 2.7 \\
\hline
\end{tabular}

Note-Frame cue $=[$ (average of algebraic deviations with frame to the right) - (average of algebraic deviations with frame to the left) $] / 2$. Head cue $=[$ (average of algebraic deviations with head to the right) - (average of algebraic deviations with head to the left $)] / 2$. Disk cue $=[($ average of algebraic deviations with disk rotating $\mathrm{CW}$ ) - (average of algebraic deviations with disk rotating $\mathrm{CCW})] / 2$. 
Table A3

Means and Standard Deviations in Degrees of the Sensitivity to Specific Disturbance Computed in Experiments 1 and 2 for Women and Men

\begin{tabular}{|c|c|c|c|c|c|c|c|c|}
\hline \multirow[b]{3}{*}{ Condition } & \multicolumn{4}{|c|}{ Experiment 1} & \multicolumn{4}{|c|}{ Experiment 2} \\
\hline & \multicolumn{2}{|c|}{ Head Cue } & \multicolumn{2}{|c|}{ Frame Cue } & \multicolumn{2}{|c|}{ Head Cue } & \multicolumn{2}{|c|}{ Disk Cue } \\
\hline & $M$ & $S D$ & $M$ & $\overline{S D}$ & $M$ & $S D$ & $M$ & $S D$ \\
\hline \multicolumn{9}{|c|}{ Women } \\
\hline Inclined head in control condition & 2.35 & 2.4 & & & 2.03 & 2.7 & & \\
\hline Head upright with visual perturbation & & & 2.34 & 1.9 & & & 3.24 & 1.9 \\
\hline Inclined head and visual perturbation & 2.65 & 4.9 & 2.9 & 2.4 & 1.05 & 2.4 & 5.5 & 2.4 \\
\hline \multicolumn{9}{|l|}{ 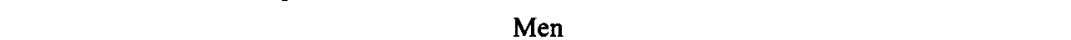 } \\
\hline Inclined head in control condition & 0.62 & 2.5 & & & 0.43 & 2.8 & & \\
\hline Head upright with visual perturbation & & & 0.99 & 1.6 & & & 1.9 & 1.5 \\
\hline Inclined head and visual perturbation & 1.03 & 4.1 & 0.1 & 1.5 & -1.2 & 3.3 & 4.05 & 2.7 \\
\hline
\end{tabular}

Note - Frame cue $=[$ (average of algebraic deviations with frame to the right) - (average of algebraic deviations with frame to the left $)] / 2$. Head cue $=[($ average of algebraic deviations with head to the right) - (average of algebraic deviations with head to the left)]/2. Disk cue $=[$ (average of algebraic deviations with disk rotating $\mathrm{CW}$ ) - (average of algebraic deviations with disk rotating $\mathrm{CCW})] / 2$.

(Manuscript received July 8, 1996;

revision accepted for publication February 12, 1997.) 